\section{Animate materials}

\section{By Philip Ball}

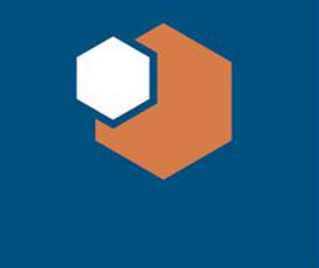

tunnel, or failure and corrosion of a biomedical implant. Making new materials and structures is typically not only a very energy-intensive process but also wasteful. Making a desktop computer consumes around 1.5 tonnes of water and $240 \mathrm{~kg}$ of fossil fuels - and if a device like this fails, it is often cheaper and easier to replace it than repair it.

Materials with "animate" properties would not only make manufacturing and engineering cheaper and more sustainable, they would alter the whole notion of what these words mean. A material that adapts its shape and structure to the environment does not have to be optimized at the outset for a given situation (with the risk that it then performs poorly if the conditions change). And a material that builds itself does not have to be painstakingly assembled before being placed. "Smart cities" of the future are likely to have to adapt to significant changes in climate: higher temperatures, more humidity, greater fluctuations and extremes. In space exploration, a selfhealing material could make the difference between mission success and failure, and possibly between life and near-instant death. Materials like this need not lose their value and be thrown away, although they will probably need to be embedded in an efficient "metabolic ecosystem" that supplies fresh raw ingredients and energy to enable the materials to sustain their function.

This article is a call for a dedicated effort to develop animate materials. These should be regarded not merely as a new class of material, but as a basis for rethinking the goals of a large segment of applied science, from civil engineering to biomedicine, driven by the urgent needs of a society facing grave economic, demographic, and environmental challenges. The concept of animate materials reflects a growing view that something needs to shift in our philosophy of making if we are to meet these challenges in the near future. can be devastating and fatal, whethe that involves collapse of a bridge or a

\section{Where do animate materials fit} in?

Ever since humans began constructing artifacts from materials - whether a stone bridge or a space rocket-we have regarded design as a fixed objective, and have selected materials and structures that we believe will do the job as well as possible. Maintenance, repair, and alteration have then been regarded as additional tasks - necessary in most cases, but enacted post hoc. Typically, the aim has been to use materials that resist change: that will not break or corrode or deform. If these things happen, a whole new train of interventions is required.

But for animate materials, responses to such changes are foreseen and accommodated from the outset. Indeed, they can even be seen as opportunities: such materials might interactively improve the original design as the constraints change (see Figure 1).

Genuinely animate materials - those found in nature - do this already (see Figure 2). Wood, shell, and skin are also able to self-heal and adapt to the prevailing conditions. There has been intense interest for many decades in making materials that can mimic some of those found in nature, thereby capturing some of their superior properties. For example, some tough ceramic composites use tricks taken from the microstructure of shells, such as mother-ofpearl, in which strong, hard plates are bonded together in stacks by softer, weaker material so that the energy of an impact is absorbed by the delamination of these layers, preventing a crack from spreading as it would through a monolithic material. This field of biomimetic materials engineering ${ }^{2}$ has produced some wonderful results, but it does not really capture what is truly remarkable about biomaterials: their adaptability and their capacity for self-assembly and self-repair. It is capabilities like those that will distinguish animate materials from the smart and biomimetic materials that have been mostly developed to date.

Allied to biomimetics is the field of biocompatible and bioactive materials, 


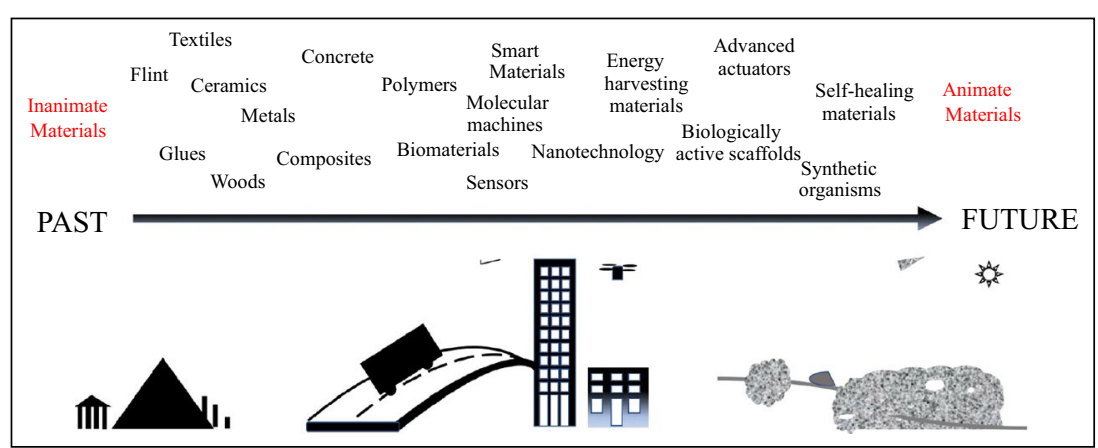

Figure 1. The direction of travel for materials since antiquity is toward increased functionality and adaptability, leading ultimately to materials with genuinely animate properties. Image: Mark Miodownik, University College London and the Institute of Making.

designed to interact favorably with the body, for example to make implants and biomedical devices that merge seamlessly with tissues. These include bioglasses that encourage bonding to bone, and polymers coated or impregnated with biological agents that assist wound healing or suppress blood clotting.

The notion of materials that respond to their environment-smart materials-is also long established. The idea of the smart material is that some environmental stimulus - a change in temperature, say, or an electrical field or mechanical vibration-alters the

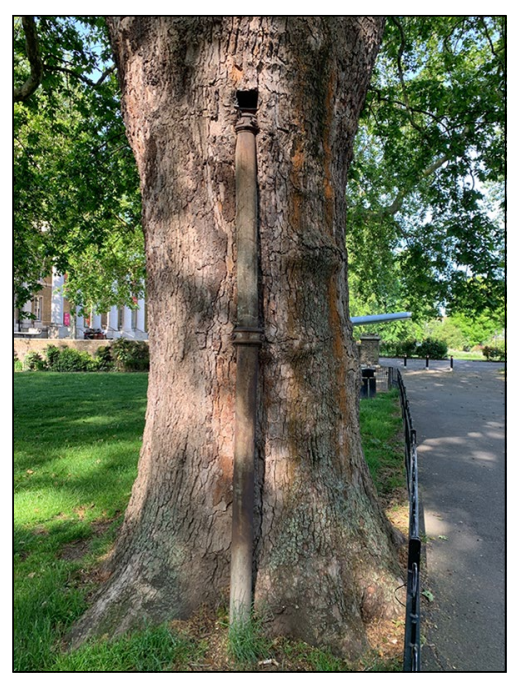

Figure 2. Adaptive, autonomous growth of a tree to accommodate human technology. Image: Ruby Wright. properties of the material in a manner that is of practical value. Photochromic glass is one of the oldest and most familiar examples: the transparent material darkens and reduces its light transmission as the light grows brighter. Materials with an adaptive mechanical pliancy could help to damp the vibrations of earthquakes. Polymers that change their shape or volume in response to electrical or chemical signals can provide parts for "soft machines": valves, pumps, filters. Thermoelectric materials generate electrical fields in response to heat, allowing energy generation from waste heat in vehicles.

Soft polymer systems have been one of the most explored fabrics for creating environmentally responsive smart materials, not least because of their potential biocompatibility. In particular, hydrogels that swell or shrink - altering their water retention through the opening or collapse of pores and cavities between cross-linked strands-in response to changes in temperature or $\mathrm{pH}$ have been used for controlled drug delivery and mechanical actuation. Smart gels and shape-memory polymers, which can be switched reversibly between different macroscopic shapes, have been used in conjunction with threedimensional (3D) printing technology to make biocompatible "4D" devices with programmed mechanical behavior, the fourth dimension here referring to the dynamical degrees of freedom. ${ }^{3}$
Often these gels are held together by noncovalent interactions, which means that they will reform and "heal" if disrupted. Aizenberg and co-workers have achieved multi-directional mechanical responsiveness in an elastomeric system. They incorporated light-sensitive isomerizable chemical units into liquidcrystalline polymers, using the molecular orientation of such building blocks to make a rubbery material that will flex in the direction of illumination. This is rather like the way some plants will flex their stems so as to keep their heads pointed toward the sun.

Smart materials are, however, generally designed for a single, specific function. The functionality may be expanded by incorporating them in smart composite systems with, for example, sensing, actuating, and computing fuctionality. ${ }^{4}$ But in general such systems are composed of many parts and devices, integrated in an assembly in the manner of robotic engineering. Animate materials are likely to be inherently multifunctional: they might display more versatility and, one might say, more intelligence, within a single component or unit.

Self-healing looks set to become an almost paradigmatic property of animate materials. It is in fact a capability that has been exercised for millennia, although not by design. The lime mortar used since ancient times to bond bricks and stones in building structures is a mixture of calcium hydroxide (lime), sand, and water that solidifies into a hard ceramic. But if it cracks, moisture entering into the fracture can activate unreacted lime to crystallize and seal up the crack. This is why walls and bridges built many centuries ago have been able to withstand changes in stress loading, for example caused by earthquakes, sometimes rather better than buildings constructed with newer mortar formulations that lack this self-healing capacity.

These old mortars essentially contain a reservoir of the material needed to heal cracks. These can be built into artificial materials by design, creating self-healing materials that may find applications in construction of roads, 
buildings, aircraft, and space technologies. The need for such materials is plain enough: it is estimated that repair and maintenance of the (mostly concrete) building infrastructure of the UK costs around $£ 40$ billion a year, and about half of such repairs fail after just 10 years.

Self-healing concretes may contain capsules filled with some agent that, when released from a ruptured capsule, promotes chemical reactions that seal a crack. Typically the capsules are made of soft polymers (such as gelatin or gum arabic) filled with epoxy adhesive or sodium silicate, which mineralizes on exposure to air. ${ }^{5}$ Here healing is a single-shot affair, but repeat healing can be achieved by introducing vascular-like networks along which the healing agent can be injected: for example, created by polymer tubes that act as molds for the channels and which are removed once the concrete has hardened.

One dramatic approach to self-healing concrete exploits the responsiveness of living systems themselves. The concrete incorporates spores of bacteria able to withstand the highly alkaline conditions that exist in concrete manufacture, and which excrete a mineral (calcite) that is itself a component of concrete. The bacteria lie dormant in the material until a crack exposes

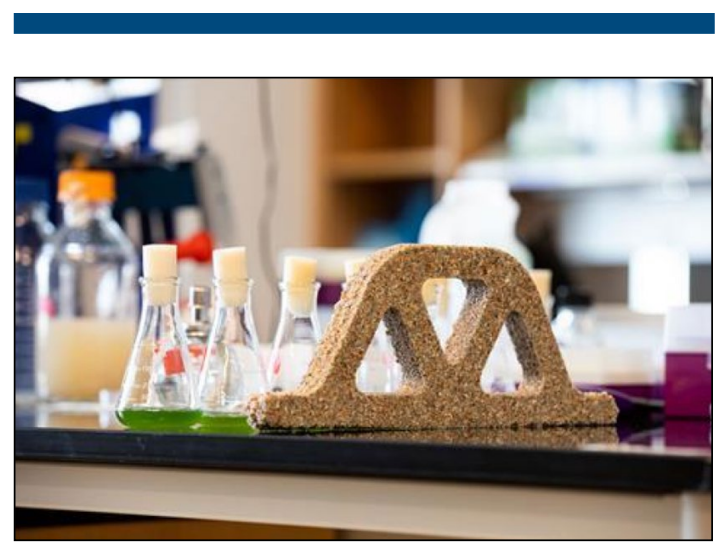

Figure 3. A "living" cement-like structure made from a sand-hydrogel composite permeated with biomineralizing green photosynthetic cyanobacteria. The structure is able to regenerate when subdivided, if provided with the inorganic raw materials. From Reference 6; image courtesy of University of Colorado Boulder, College of Engineering and Applied Science. them to air and moisture, whereupon they become active (fed by starch also added to the concrete formulation) and make calcite - which can seal the crack and restore most of the concrete's strength. These approaches have performed promisingly in field trials but have not yet been used in civil structures. Recently this approach of permeating an inorganic cement-like matrix with biomineralizing microorganisms has been used to make "living bricks" that show a primitive form of regeneration and growth, being able to "reproduce" over several generations (see Figure 3$){ }^{6}$

An analogous approach of releasing stored healing agents has been pursued with rubbery polymers: the rupture of small embedded capsules can release chemical agents that will mix and react to "cure" and polymerize them, again creating a self-healing adhesive. Again, moving beyond single-use approaches in polymers, composites, and other materials systems has so far involved vascular networks of channels to introduce fresh reagents on demand.

Other self-healing polymers rely on an ability to reconstitute themselves when damaged, thanks to factors such as intrinsic polymer chain mobility and entanglement, polymerization reactions, ${ }^{7}$ or weak interactions such as hydrogen-bonding or electrostatic attractions (in ionically cross-linked ionomers, say) that can break and reform via supramolecular interactions. A self-healing polyurethane coating incorporating chemical groups related to chitin has been developed that exposes reactive groups when mechanically damaged that will repair in less than an hour on exposure to ultraviolet light. The necessary flow or plasticity might be triggered by some external stimulus such as light or heat. One of the major drivers for selfhealing polymeric paints and coatings is the automotive industry: the demand for vehicle paints in which polymeric binding agents can flow and cover small scratches and flaws in bodywork is obvious.

One particularly attractive application of self-healing materials would be for road repair. Potholes in roads pose serious hazards for vehicles, and their repair - patchy at best - costs the UK around $£ 4$ billion a year. Asphalt used for road surfacing is a mixture of rubble-like aggregate with a binder of ultra-viscous bitumen. This has some natural self-healing capacity, as bitumen will drain slowly into microcracks. But the process takes several days because bitumen is so viscous. Crack growth rate can outstrip healing, and big cracks open up. Two main solutions are being explored. ${ }^{8}$ First, drainage can be accelerated by local heating of the asphalt to make the bitumen more fluid, using microwaves, infrared radiation or "induction heating" in which electromagnetic fields induce currents and resistive warming of buried metal fibers or particles. There are problems with such approaches, however: for example, microwaves can cause embrittlement, and are reflected by buried metal structures.

The second approach involves encapsulated oil-based solvents (typically spherical particles with a calcium alginate membrane), which reduce the bitumen viscosity when the capsules are broken by cracking and their contents are released. One problem is that solvents may leak out over time. Clearly there is much work to be done to make roads that can self-heal in an economically viable and long-term sustainable manner. The ad hoc solutions used for such systems at present, although potentially effective, often demand complicated and costly human interventions and do not really achieve the full potential of making materials animate. 


\section{A taxonomy of materials}

While it is probably not helpful to seek rigid demarcations or definitions of what is "smart," "biomimetic," "animate," and so forth, situating a research effort on animate materials would be aided by having a clearer view of where the target lies in the space of possible materials.

One perspective would be to equate "animate" with an autonomous capacity to "act"- which is reflected in the perception that many of the properties considered desirable in animate materials are prefixed with "self-": assembly, repair, sensing, diagnosing, reporting, protecting. In general, the aim is to make these intrinsic aspects of the materials system itself, not capabilities supplied by some external computational network or sensing mechanism: to avoid the complications and potential fragilities associated with such (typically electronic-based) designs. The material is the mechanism.

One possible way to visualize the target space is in terms of three separate axes: active, adaptive, and autonomous. "Active" means that the materials can dynamically alter their shape or properties in a goal-oriented manner by drawing on some energy source (that is, not as a merely passive response to, say,

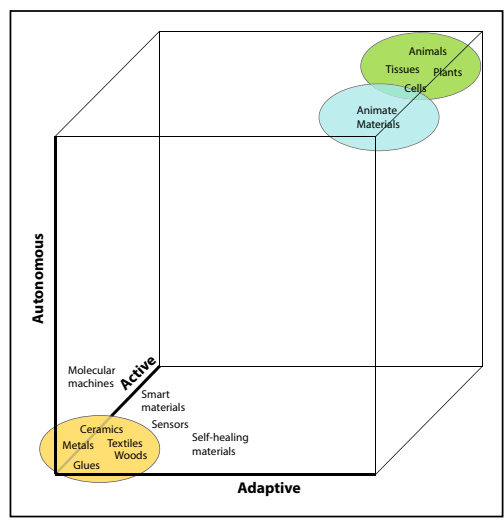

Figure 4. A proposed classification scheme for animate materials, according to the three properties of "active," "adaptive," and "autonomous." Some existing synthetic materials and systems show some of these properties, but not alland none to the extent of living materials, which exemplify the part of this design space we need to move toward. thermal activation). "Adaptive" means that they can sense and respond to their environment. "Autonomous" means that they can decide or compute their response from the input signals.

Animate materials, like living systems, score highly in all three respects, as do "living technologies" such as tissue culture that already make use of living systems for technological ends. Traditional materials such as metals and ceramics lie close to the origin in this space, while current smart materials are somewhat active but not very adaptive (except in a single capacity) or autonomous. Current self-healing materials are adaptive but often rather passive and lacking in autonomy.

Such a conceptual scheme is far from perfect, but a delineation of the "dimensions" of being animate might help to define a roadmap for research and clarify what needs to be added to existing materials technologies to bring them toward this goal (see Figure 4).

\section{Principles of animate materials}

Current self-healing materials are not actually animate, though we might regard them as "proto-animate." Their response to damage is passive, and typically one-off: an injection of adhesive from an internal reservoir. Introducing vasculature and a sensing system can make this response repeatable and more active, but at the cost of greater complication in design.

To go further may require a more generalized notion of the principles on which "animation" of materials might be based. This is not to imply that there is likely to be a single theoretical or conceptual framework for designing such materials, but rather that it will be helpful to abstract the requirements in a systematic way.

Living systems are in some respects the exemplars, but this does not mean that they should be slavishly copied. After all, they depend on a fundamental unit of immense complexity, well beyond our current capabilities to mimic: the cell. Evidently the cell does a great deal more than is strictly needed to make a material with anything approximating the animate nature of bone: it can replicate, move, metabolize, and self-destruct, for example. Perhaps the key questions that living systems pose for a generalized scheme of animate materials are as follows:

- Do such materials need to be based on a modular structure of discrete, autonomous units?

- Where in the system is the information stored and transmitted that enables it to do its job?

- Will these systems have to be active, in the sense of requiring an energy source?

It is striking that some of these questions have been explored already in one approach to the synthesis of adaptive materials systems, sometimes called programmable matter (or "smart dust"). ${ }^{9}$ The concept here is really a kind of miniaturization of trends in robotics, where multivalent, adaptive robots are designed in modular fashion so that the units are reconfigurable. A central question here is how much autonomy to give each unit: do they decide collectively how to assemble themselves, based on interactions between them and with the environment, or would they be programmed and controlled by some external agency? How much of the resulting behavior, in other words, is emergent (bottom up), and how much comes from top-down control?

At present, it remains very challenging to create tiny mechanical structures with adequate faculties for sensing, movement, and decision making; smart dust and nanotechnological self-assemblers remain very much in the realm of fiction. But miniature robots capable of assembling components into complex structures have been demonstrated, and have been proposed for construction in extreme environments such as human space exploration. Such systems might build not according to some predetermined plan but in a manner that responds to the surroundings, including the presence of other robots. This would be a type of self-assembly allied 
to cellular automata models, which have been explored as simple prototypes of living systems made from cells, as well as of the way some insect colonies build their nests. Their collective behavior might deliver robust solutions even in the face of occasional failure of the agents because of redundancy: one agent can take over the task of another.

Such a perspective also raises the question of whether there might be some fundamental toolkit or catalogue of parts from which many materials configurations might be constructedalong the lines of the standardized components (e.g., diodes, resistors, and transistors) of electronic circuitry, or the "BioBricks" or Registry of Standardized Parts envisaged for synthetic biology. Researchers at the Massachusetts Institute of Technology's Center for Bits and Atoms are exploring the notion of developing a small set of mechanical components for building more or less any robotic or actuating structure.

One risk in such systems is overdesign: the agents might be far more complicated than they really need to be to achieve a specific set of goals, and the space of possible outcomes correspondingly larger than needed. To develop good design principles for making animate materials, we could be guided by a better picture of what "animate" means in biology.

One of the most obvious molecularscale differences between living and inanimate materials is the nature of the chemical bonding. Rocks and minerals tend to have rigid structures held together by strong (covalent or ionic) chemical bonds. In living systems, the molecules - such as proteins and nucleic acids - are generally dynamic in their conformation and interact with other molecules via weak interactions such as hydrogen bonding and hydrophobic forces. This enables information to be transmitted from place to place, and means that molecular entities can be assembled and disassembled on demand. The "looseness of structure" is surely also an essential feature for systems that are maintained away from equilibrium by a throughput of material and energy, and which can adapt to changes in the environment.

It seems likely that many animate materials will use the same principles. There is now a long tradition of molecules designed to self-assemble into clusters, crystals, and materials via the judicious placement of mutually interacting groups. Molecular machines rely, ${ }^{10}$ like their biological counterparts such as motor proteins, on the ability of molecules to switch conformation in response to some stimulus, such as light, ions, or chemical "fuel." Switchable components like this can be selfassembled into extended arrays to create materials with programmable structures and mechanical behavior.

DNA itself is proving to be an extremely versatile substrate for the synthesis of machines and structures of this sort, by taking advantage of the programmable specificity with which complementary single strands will pair up. ${ }^{11}$ With the help of computer algorithms to design the requisite sequences, artificial DNA strands can be programmed to assemble into more or less any arbitrary structure, including 2D and 3D arrays. This informational aspect of DNA, which of course is what makes it the molecular carrier of inherited genetic information, makes it the perfect exemplar of Jean-Marie Lehn's concept of "informed matter." It can also be used to store information at extremely high density, creating not just new types of molecule-based memories but also materials that could carry the instructions for their own performance. DNA constructs have been shown to be capable of computation, for example by self-assembly of "tiles" that act like cellular automata.

The use of DNA also makes such materials potentially compatible with living matter, and able to enter into dialogue with it. At the same time, extensions of the genetic code to add nonnatural "letters" mean that DNA-based devices and materials could be rendered orthogonal to living systems, coexisting without interfering or becoming targets for degradation. Artificial DNA constructs that process and actuate input signals can be encapsulated inside synthetic "protocells" to create "informed" microscale entities that take cues from nature without merely recapitulating its molecule mechanisms.

Not all self-assembly and selforganization processes in cells depend on such specificity of weak molecular interactions. Forces lacking the directionality of hydrogen bonds or coordination bonds, such as van der Waals and hydrophobic interactions, can also drive the formation of structure in a manner that can be reversed and reconstituted.

Phase-separation processes have also now been found to be important in cell biophysics, for example leading to the formation of non-membrane-bound organelles. When they are constrained to happen on molecular length scales, for example in block copolymers, this can lead to the appearance of ordered microphases with useful and tunable mechanical properties. Stupp and coworkers have used similar processes of molecular aggregation to make peptidebased amphiphilic polymers that will spontaneously assemble from solution into fibrous and other structures, making them biocompatible materials that could be injected to form scaffolds for wound healing and other physiological processes in situ. ${ }^{12}$

Colloidal particles, typically on the scale of micrometers, will self-assemble into loose crystals. These can have useful optical properties, for example creating colored materials through selective light-scattering. Such selfassembly processes happen in nature, as for example in the formation of iridescent opal, a biomineral. Ordered biological structures like this, with periodicities of the same order as the wavelengths of visible light, have been found to act as natural optical fibers in sea sponges and worms and as sources of coloration in butterfly wings. Colloidal assembly can be driven by entropic forces, ${ }^{13}$ which, guided by geometric factors, can give rise to complex structures. Because only relatively weak forces bind them, they might be disassembled and reassembled for colloidal "entropic robotics." 
There has been a great deal of interest in the past decade or so in colloidal-scale particles that have their own source of motion, for example because of chemical reactions on their surface or in response to magnetic fields. These systems are known as active matter, ${ }^{14}$ and they can exhibit complex forms of dynamic self-organization reminiscent of the behaviors of some insect or bacterial colonies. These modes of organization can be sensitive to external conditions, and can offer ways to imbue materials systems with dynamic responsiveness.

\section{Toward protocell materials}

One of the key features of living systems is that they involve a hierarchical cascade of self-organization processes on different length scales. This is not (contrary to popular belief) a scheme fully programmed into the system at the smallest scale (i.e. the DNA sequence of a genome) that is then systematically read out like a blueprint. Rather, each level of the assembly process establishes the basic elements of the next, which then interact according to new rules, based on physical chemistry, that dictate the next level of emergent structure. One might consider this process to be iterated all the way from the genome to the organism and beyond: to colonies, communities, and social systems - and to be refined and elaborated by evolution.

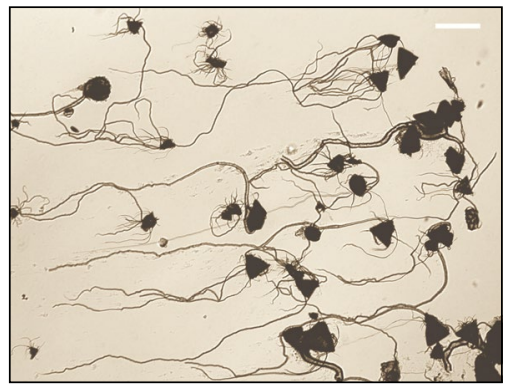

Figure 5. Complex precipitation structures grown from polyoxometalates, with morphologies resembling those of biological growth. Image: Lee Cronin, University of Glasgow.
Animate materials might mimic this idea without slavishly copying it. It is an open question how "designable" a scheme it is; it may be that there must be an element of trial and error, of exploring the landscape of possible outcomes from a basic set of building blocks, perhaps guided by in vitro selection, mutation, and evolution. For example, a classic chemical paradigm for creating complex macroscale structures from simple, self-organizing ingredients is the "chemical garden" in which silicate ions assemble in solution into semi-permeable membranes that become extended into tubular and branching structures with an "organic" appearance. Cronin and co-workers have shown that such forms can grow also from polyoxometalates with some control of the growth morphologies (see Figure 5). ${ }^{15}$ It has been suggested that structures like these, growing in the solute-rich environment of hydrothermal vents in the deep ocean, might have been involved in the origin of life: the inorganic membranes create conditions for chemical gradients that can be tapped as an energy source for primitive metabolic processes, while closed membranes budding from such tubes could act as protocells.

Such studies demonstrate that behavior reminiscent of living organisms can arise from simple inorganic ingredients, without any need for the complex organic species such as proto-peptides and nucleic acids that have typically been the object of prebiotic chemical studies. This consideration prompts suggestions that, to create proto-living artificial systems, we should not focus too much on the precise chemical character of the components but should think instead about how nonequilibrium physics might offer general mechanisms for the formation of self-sustaining and self-replicating structures. The key issue is not so much the molecules themselves but the nature of the interactions between them: how they might organize into autocatalytic networks that can give rise to a primitive form of replication and metabolism.
How far animate materials might need to make use of such complex behaviors is open to question, but they are amenable to design. Parrilla-Gutierrez and colleagues have, for example, reported a robotic fluidic system that can evolve the composition of oil-based surfactant protocell droplets via the selective pressure imposed by altering the environmental conditions. ${ }^{16}$ Robotics combined with artificial intelligence methods can permit a systematic exploration of the complex behaviors such systems can show, including droplet division, movement, and collective swarming.

Ultimately, the key characteristic that might distinguish a genuinely "animate" nature in a material might be expressed as "agency": a capacity for internal, autonomous control, in a manner that goes beyond an automatic, passive response to a stimulus and which involves some kind of decision-making process in which an output is computed from inputs. There is currently no welldeveloped theoretical description of agency-but if such a thing exists, it is likely to follow from an abstraction of the specific mechanisms by which these qualities are developed in living systems so as to capture the principles that might be reproduced in nonliving materials. These are likely to include (but not limited to) memory and representation of the environment, energy capture from the environment, adaptation to prevailing circumstances, compartmentalization and structuring of the physical space of the system, and the harnessing of nonequilibrium dynamics.

Experiments on "protocells" made from nonbiological components might point to a bottom-up, and perhaps even evolutionary, route to these capabilities. Mann and colleagues have explored the synthesis of liquid-droplet protocells called coercevates, made by the association of two kinds of macromolecule or material with opposite electrical charge (such as DNA and clay particles). The lack of a membrane in such droplets can be an advantage, as it enables them to engulf one another, divide, and reform. 
Mann and colleagues have made DNA/ clay protocells containing enzymes that can control their buoyancy by the catalytic production of oxygen, and polymeric "protocell" particles capable of self-assembling into artificial tissues that will swell and shrink in response to changes in heat because of the polymer's "smart-gel" thermally induced expansion and contraction. ${ }^{17}$

Adding membranes for compartmentalization broadens the possibilities. Membranes establish barriers to diffusion and thus can sustain energy-storing concentration gradients, and they can also supply an active platform for hosting sensing and signaling molecules, as they do in real cells.

\section{Interfaces: The need for collaboration}

While materials systems like those discussed above recapitulate some aspects of living systems, it may be that some animate materials will incorporate or interface with actual living entities. The aforementioned self-healing "bacterial cement" is one such example. The uses of synthetic biology - the manipulation and design of living cells for technological endsfor materials science have tended so far to focus on retooling cells to produce materials and their precursors, but increasingly the approach seeks to incorporate the cells themselves into functional materials.

The engineering of living matter is itself an old dream, but new technologies are finally making it a reality. The use of 3D printing with living cells ("bio-inks") makes it possible to imagine synthesizing cell-by-cell not only artificial tissues and organs but also "living" structures that do not attempt to mimic natural ones at all, but which might create new combinations of cells to produce entirely novel forms of artificial tissue. Such efforts will need a better understanding of how cells spontaneously self-organize and differentiate in response to signals from their neighbors: synthetic constructs like this will, to some degree, have a "life of their own" rather than necessarily remaining in the states and configurations in which they are laid down. ${ }^{18}$

Advances like this raise unprecedented questions about the very plasticity of living matter itself. Can aggregates of living cells support configurations that look nothing like those in nature? Might there even be entirely novel, viable, and stable cell states that are not realized in extant organisms, perhaps embodying "evolutionary memories" (as in the way mouse stem cells can form gall bladders even though mice themselves do not possess that organ)?

Another avenue for using living matter in materials contexts is to find ways of interfacing natural cells and tissues with synthetic materials. This is already the objective for the biocompatible materials mentioned earlier for implants, but there is much interest in making active interfaces in which the synthetic and living components influence one another's behavior-in particular, interfacing neurons and inorganic informationprocessing networks. Such neuralinterface technologies are of obvious interest for developing prostheses such as mechanical limbs controllable by their wearer. These already exist, but not yet as an intimate intergrowth of neural and inorganic matter: rather, they employ sensors at the interface to convert neural signals into electrical ones. Developing interfaces that use electrical, chemical, or optical signals to record and manipulate neuronal activity represents one of the major frontiers in current neuroscience. ${ }^{19}$

Possibilities like these illustrate why a community seeking to develop animate materials will need to be very broad, encompassing cell biologists and biophysicists as well as ceramicists and structural engineers, electrical engineers, and polymer chemists. It will be vital too for the research to be guided by a sound awareness and sensitivity to actual social needs, and to be cognizant of how such a change in materials philosophy might impact society in terms of, say, healthcare, environment and pollution, economic drivers (will this spell an end to "planned obsolescence"?), manufacturing, transport, and social equity. The goals of the field mean that input from social sciences and ethicists would not be an optional add-on but a key part of the vision.

\section{References}

1. Animate Materials (The Royal Society, London, UK, 2021). https://royalsociety.org/-/media/policy/projects/ animate-materials/animate-materials-report.pdf

2. P. Fratzl, J.W.C. Dunlop, R. Weinkamer, Eds., Materials Design Inspired by Nature (Royal Society of Chemistry, London, UK, 2013)

3. N.J. Castro, C. Meinert, P. Levett, D.W. Hutmacher, Curr. Opin. Biomed. Eng. 2, 67 (2017)

4. M.A. McEvoy, N. Correll, Science 347, 1328 (2015) 5. A. Al-Tabbaa, B. Lark, K. Paine, T. Jefferson, C. Litina, D. Gardner, T. Embley, Proc. Inst. Civ. Eng. Smart Infrastruct. Constr. 171 (2018), pp. 67-76

6. C.M. Heveran, S.L. Williams, J. Qiu, J. Artier, M.H. Hubler, S.M. Cook, J.C. Cameron, W.V. Srubar III, Matter 2, 481 (2020)

7. M.W. Urban, Nat. Chem. 4, 80 (2012)

8. A. Garcia, E. Schlangen, M. Van de Ven, Key Eng. Mater. 417-418, 572 (2010)

9. P. Ball, IEEE Spectr. (May 27, 2014). https://spectrum. ieee.org/robotics/robotics-hardware/make-your-ownworld-with-programmable-matter

10. V. Balzani, M. Gómez-López, J.F. Stoddart, Acc. Chem. Res. 31, 405 (1998)

11. N.C. Seeman, Nature 421, 427 (2003)

12. M.P. Hendricks, K. Sato, L.C. Palmer, S.I. Stupp, Acc. Chem. Res. 50, 2440 (2017)

13. D. Frenkel, Nat. Mater. 14, 9 (2015)

14. S. Ramaswamy, Annu. Rev. Condens. Matter Phys. 1, 323 (2010)

15. L.J. Points, G.J.T. Cooper, A. Dolbecq, P. Mialane, L. Cronin, Chem. Commun. 52, 1911 (2016)

16. J.M. Parrilla-Gutierrez, S. Tsuda, J. Grizou, J. Taylor, A. Henson, L. Cronin, Nat. Commun. 8, 1144 (2017)

17. P. Gobbo, A.J. Patil, M. Li, R. Harniman, W.H. Briscoe, S. Mann, Nat. Mater. 17, 1145 (2018)

18. R.D. Kamm, et al., APL Bioeng. 2, 040901 (2018) 19. J.A. Frank, M.-J. Antonini, P. Anikeeva, Nat. Biotechnol. 37, 1013 (2019)

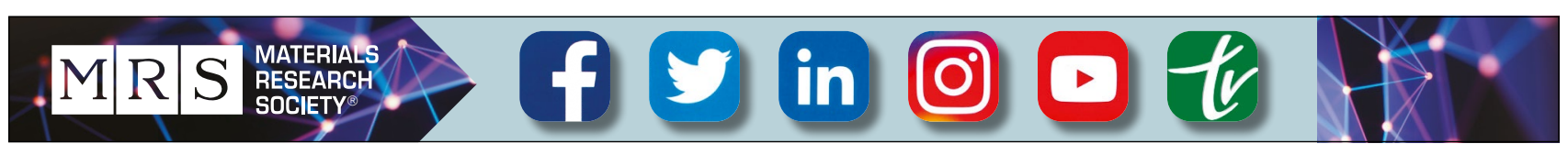

